\title{
IQGAP1 modulates the proliferation and invasion of thyroid cancer cells in response to estrogen
}

\author{
DONGDONG MENG ${ }^{1}$, WENXUN WU ${ }^{1}$, ZHIFU LI $^{2}$ and GUIJUN QIN ${ }^{1}$ \\ Departments of ${ }^{1}$ Endocrinology and ${ }^{2}$ Orthopaedic Surgery, The First Affiliated Hospital \\ of Zhengzhou University, Zhengzhou, Henan 450052, P.R. China
}

Received October 10, 2014; Accepted May 22, 2015

DOI: $10.3892 / \mathrm{ijmm} .2015 .2232$

\begin{abstract}
Thyroid cancer is an endocrine malignancy with a high incidence rate, which is affected by female hormones, particularly estrogens, in its growth and progression. IQ-domain GTPase-activating protein 1 (IQGAP1) is overexpressed in a range of types of cancer and is reported to interact with estrogen receptor $\alpha(\mathrm{ER} \alpha)$ in breast cancer cells. However, the association between IQGAP1 and ER $\alpha$ in thyroid cancer cells remains to be elucidated. In this study, the role of IQGAP1 in thyroid cancer cells was examined. The expression of IQGAP1 (190 kDa) was analyzed using western blot analysis, which indicated that IQGAP1 was overexpressed in thyroid cancer tissues and FTC133 cells. However, IQGAP1 knockdown in the FTC133 cells led to a significant downregulation in ER $\alpha$ transcriptional activity, cell proliferation, cell adhesion and cell invasion under 17 $\beta$-estradiol (E2) conditions. Furthermore, ER $\alpha$ knockdown inhibited the enhanced protein expression levels of phosphorylated ERK1/2 and cyclin D1, which were induced by the overexpression of IQGAP1. Co-immunoprecipitation wasalso performed in thyroid cancer cells and the results suggested that IQGAP1 directly interacted with ER $\alpha$ in the FTC133 cells and the co-transfected COS-7 cells. Taken together, these findings revealed that IQGAP1 may directly interact with ER $\alpha$ and serve as a signal integrator, mediating ER $\alpha$ transcriptional activity, cell proliferation and cell invasion during the progression of thyroid cancer.
\end{abstract}

\section{Introduction}

Thyroid cancer is a relatively common type of cancer associated with the endocrine system, and has increased in incidence during the past 10 years; with premenopausal women at highest risk for papillary and follicular thyroid carcinoma, implicating the role of estrogens in thyroid cancer (1) and suggesting a

Correspondence to: Dr Guijun Qin, Department of Endocrinology, The First Affiliated Hospital of Zhengzhou University, 1 Jianshe East Road, Zhengzhou, Henan 450052, P.R. China

E-mail: junqinwing@163.com

Key words: IQ-domain GTPase-activating protein 1, estrogen receptor $\alpha$, interaction, thyroid cancer, E2 specific role for $17 \beta$-estradiol (E2) stimulation and estrogen receptor (ER) expression in thyroid tumorigenesis. There are two different isoforms of nuclear estrogen receptors in humans, $\mathrm{ER} \alpha$ and ER $\beta$, together mediating the role of estrogens. They have several similarities in structural and functional domains, however, they differ in tissue distribution and function $(2,3)$. In general, ER $\alpha$ is involved in a series of cellular processes, including cell proliferation, invasion, differentiation and apoptosis (2), whereas E2 acts in the target cells through binding to the ligand-binding domain of ER $\alpha$. Notably, dysfunction of the estrogenic system is often associated with the progression of several types of lesion, including cancer (4).

IQ-domain GTPase-activating proteins (IQGAPs) are an evolutionary conserved family containing multi-domain proteins, which have been identified in numerous organisms ranging from yeasts to mammals. Of the IQGAP proteins, IQGAP1, IQGAP2 and IQGAP3, have been confirmed to be ubiquitously expressed in humans and involved in multiple cellular processes, including cell adhesion, cell migration, extracellular signaling and cytokinesis (5-7). The most extensively investigated family member is the scaffold IQGAP1 protein, which is involved in multiple essential biology processes by binding to numerous interacting proteins and mediating their functions (8). Increasing evidence has emerged to suggest that IQGAP1 may contribute to the progression of several types of cancer, including thyroid cancer, lung cancer, ovarian cancer gastric cancer, hepatocellular carcinoma, breast cancer and melanoma (9-13). In addition, IQGAP1 knockdown attenuates the transcription ability of estrogen-responsive genes, induced by estrogen via binding to $\mathrm{ER} \alpha(4)$, and the overexpression of IQGAP1 has been associated with the invasiveness of thyroid cancer cells (14).

In addition, the extracellular signal-regulated kinase (ERK) pathway, a mitogen-activated protein kinase (MAPK) pathway, is known to be activated by receptor tyrosine kinases, cytokine receptors, and G-protein-coupled receptors $(15,16)$. In particular, the phosphorylation of certain target proteins by ERK1/2 is involved in transcriptional activation by coordinating extracellular cues and intracellular signals $(15,16)$. Previous studies have shown that IQGAP1 modulates the ERK pathway in certain cells and can also function independently of this mechanism (2-4), and that $\mathrm{ER} \alpha$ is a major driver of growth in tamoxifen-resistant breast cancer cells, supported by human epithelial growth factor receptor (HER)/ERK signaling (17). This indicates that IQGAP1 and ER $\alpha$ are associated with the ERK signaling pathway in cancers. 
The present study aimed to investigate how the overexpression or knockdown of IQGAP1 in thyroid cancer cells alters cell proliferation, in particular cell invasion, as well as the ERK pathway, thus identifying the physiological functions of IQGAP1. In addition, the effect of IQGAP1 knockdown on the transcriptional activity of ER $\alpha$ and the IQGAP1/ER $\alpha$ interaction were evaluated in vitro using co-immunoprecipitation and protein-binding assays, to investigate their role in the proliferation and invasion of thyroid cancer cells.

\section{Materials and methods}

Tissue samples and cell culture. Follicular thyroid cancer tissue samples $(n=10)$ and adjacent non-neoplastic tissue samples $(n=10)$ were originally obtained from patients at the First Affiliated Hospital of Zhengzhou University with informed consent at the time of surgery in accordance with the Declaration of Helsinki. The 10 patients were females with a mean age of 41.4 years (SD, \pm 8.9 years) and were diagnosed with poorly differentiated follicular cancer. The tissue samples were then prepared for reverse transcription-quantitative polymerase chain reaction (RT-qPCR) and western blot analyses. This study was approved by the Ethics Committee of Zhengzhou University (Zhengzhou, China). The FTC133 human follicular thyroid cancinoma cell line and the Nthy-ori 3-1 normal thyroid follicular epithelial cell line were obtained from the European Collection of Cell Cultures (Wiltshire, UK). The simian-derived COS-7 cell line was purchased from American Type Culture Collection (Manassas, VA, USA). The FTC133 cell line was maintained in Dulbecco's modified Eagle's medium (DMEM; Gibco Life Technologies, Rockville, MD, USA)/F12 medium. The Nthy-ori 3-1 cell line was maintained in RPMI-1640 medium (Gibco Life Technologies). The COS-7 cell line was maintained in DMEM. The three cell lines were equally supplemented with $10 \%$ fetal bovine serum (FBS; Gibco Life Technologies), 2 mM L-glutamine, $100 \mathrm{U} / \mathrm{ml}$ penicillin and $100 \mu \mathrm{g} / \mathrm{ml}$ streptomycin (Gibco Life Technologies), in a humidified atmosphere of $5 \% \mathrm{CO}_{2} / 95 \%$ air at $37^{\circ} \mathrm{C}$.

RNA extraction and RT-qPCR analysis. Total RNA was extracted from the FTC133 cells using TRIzol reagent (Invitrogen Life Technologies, Carlsbad, CA, USA), according to the manufacturer's instructions. Reverse transcription was performed with $1 \mu \mathrm{g}$ total RNA using the high capacity cDNA reverse transcription kit (Applied Biosystems, Foster City, CA, USA). The gene sequences encoding the human IQGAP1 and ER $\alpha$ full-length molecules were then amplified by qPCR. Amplification was performed using a Takara Ex Taq PCR kit (Takara Biotechnology Co.,Ltd., Dalian, China) and conducted in a $50 \mu \mathrm{L}$ reaction volume containing $5 \mu \mathrm{l}$ cDNA, $1 \mathrm{U}$ Ex Taq polymerase, $50 \mathrm{pM}$ of each primer $(1 \mu \mathrm{l}), 2.5 \mathrm{mM}$ of each dNTP $(8 \mu \mathrm{l})$, and $5 \mu \mathrm{l} 10 \mathrm{X}$ PCR buffer on a I-cycler thermal cycler PCR (Bio-Rad, Hercules, CA). The following specific primers were used: IQGAP1, sense 5'-CTAGCTAGCATGTC CGCCGCAGACGAGG-3' (NheI site) and antisense 5'-CCGC TCGAGCCTCGTCTGCGGCGGACAT-3' (XhoI site); and ER $\alpha$, sense 5'-CTAGCTAGCATGACCATGACCCTCCACA CCA-3' (NheI site) and antisense 5'-CCGCTCGAGTGGTGT GGAGGGTCATGGTCAT-3' (XhoI site). The qPCR reactions were performed using the following conditions: $95^{\circ} \mathrm{C}$ for
$1 \mathrm{~min}$, and 30 cycles of $95^{\circ} \mathrm{C}$ for $1 \mathrm{~min}, 60^{\circ} \mathrm{C}$ for $1 \mathrm{~min}, 72^{\circ} \mathrm{C}$ for $2 \mathrm{~min}$, then $72^{\circ} \mathrm{C}$ for $10 \mathrm{~min}$ and $4^{\circ} \mathrm{C}$ for $5 \mathrm{~min}$. All primers specific to IQGAP1 and ER $\alpha$ were designed using Premier 5.0 software (Premier Biosoft International, Palo Alto, CA, USA). All other regents used in the assay were purchased from Takara Biotechnology Co., Ltd.

Plasmid construction of IQGAPI- and ER $\alpha$-small interfering RNA (siRNA), and IQGAPI and ER overexpression plasmids. For gene silencing experiments, IQGAP1- and ER $\alpha$ specific siRNA and negative siRNA were synthesized by Takara Biotechnology Co., Ltd. and annealed into doublestranded molecules using an siRNA construction kit (Ambion Life Technologies, Austin, TX, USA), which were then cloned into the Bam HI/ClaI sites of pSUPER-neo vectors (OligoEngine, Seattle, WA, USA). Using this method, pSUPER-neoIQGAP1-siRNA plasmids and pSUPER-neo-ER $\alpha$-siRNA plasmids were obtained. The sequences of the specific siRNA against IQGAP1 were as follows: Forward, 5'-AAGGCCG AACTAGTGAAACTGCCTGTCTC-3' and reverse, 5'-AAC AGTTTCACTAGTTCGGCCCCTGTCTC-3'. The sequences of the nonspecific (NS) siRNA control were as follows: Forward, 5'-AAGTACCAAGGACGCGAATGTCCTGTCTC-3' and reverse, 5'-AAACATTCGCGTCCTTGGTACCCTGT CTC-3'; the sequences of specific siRNA against ER $\alpha$ were as follows: Foward, 5'-CCTCGGGCTGTGCTCTTTTTTCC TGTCTC-3' and reverse, 5'-AAAAGAGCACAGCCCGAGG TTCCTGTCTC-3'; the sequences of the NS siRNA control were as follows: Forward, 5'-AATTCTCCGAACGTGTCA CGTCCTGTCTC-3' and reverse, 5'-AAACGTGACACGTT CGGAGAACCTGTCTC-3'.

In addition, pcDNA3.1-IQGAP1 and pcDNA3.1-ER $\alpha$ were constructed by inserting the full-length coding region of the IQGAP1 and ER $\alpha$ cDNA into the NheI/XhoI sites of the pcDNA3.1 (Invitrogen Life Technologies), respectively, which were finally identified by enzyme digestion and sequencing. The constructed plasmids were digested with the restriction enzymes, NheI and XhoI (Takara Biotechnology Co., Ltd.), at $37^{\circ} \mathrm{C}$ for $4 \mathrm{~h}$ and then sequenced by Takara Biotechnology Co., Ltd.

Transfection with the expression vector. The cultured cells were seeded in 6 -well plates at a density of $4 \times 10^{5}$ cells/well and transfected with a constructed vector, as described above, using Lipofectamine 2000 (Invitrogen Life Technologies), according to the instructions of the manufacturer. The pSUPER-neo-IQGAP1-siRNA or pSUPER-neo-IQGAP1-siRNA-N plasmids, and the pSUPER-neo-ER $\alpha$-siRNA or pSUPER-neo-ER $\alpha$-siRNA-N plasmids were transfected into the FTC133 cells for detection of the function of IQGAP1. The pcDNA3.1-IQGAP1 plasmids and pcDNA3.1-ER $\alpha$ plasmids were co-transfected into COS-7 cells or FTC133 cells cells at a density of $4 \times 10^{5}$ cells/well in 6-well plates for in vivo protein-protein interaction assays. The transfected cells were then exposed to $500 \mu \mathrm{g} / \mathrm{ml}$ Geneticin (G418 sulfate; Invitrogen Life Technologies) for $\sim 3$ weeks for the formation of G418-resistant colonies at $37^{\circ} \mathrm{C}$. To ascertain the specificity of the siRNAs and expression vector used, the mRNA and protein levels, as well as the effects on the functional targets, of the IQGAP1 and ER $\alpha$ proteins were monitored by RT-qPCR, western blot analysis and reporter luciferase assay. 
Western blot analysis. As regards the fresh tissue samples, $\sim 500 \mathrm{mg}$ of frozen thyroid tissue was sectioned into small sections, homogenized and dissolved in $500 \mu 1$ RIPA lysis buffer (Beyotime Institute of Biotechnology, Shanghai, China). The cultured FTC133, Nthy-ori 3-1 and COS-7 cells were extracted, as indicated, following transfection and treatment. The protein concentrations of the samples were determined using a Bradford assay, and equal quantities of protein were loaded onto a sodium dodecyl sulfate-polyacrylamide gel electrophoresis (SDS-PAGE) gel (Shanghai Sangon Biotechnology Co. Ltd, Shanghai, China), prior to being transferred onto polyvinylidene difluoride membranes (PVDF; Millipore, Bedford, MA, USA). Subsequently, the membranes were blocked with $5 \%$ non-fat milk in $0.1 \%$ Trisbuffered saline with Tween-20 (TBST; Shanghai Sangon Biotechnology Co. Ltd.) for $1 \mathrm{~h}$ at room temperature, followed by incubation with the primary antibodies overnight at $4^{\circ} \mathrm{C}$. The antibodies used were mouse anti-human IQGAP1 monoclonal antibody (\#610611) or mouse anti-human E-cadherin monoclonal antibody (\#610181) (dilution, 1:2000; BD Biosciences, San Jose, CA, USA); mouse anti-human ER $\alpha$ monoclonal antibody (sc73479), rabbit polyclonal IgG anti-p-ERK1/2 antibody (sc16981-R), mouse monoclonal IgG anti-ERK1/2 antibody (sc-135900), mouse anti-human matrix metalloproteinase-9 (MMP-9) monoclonal antibody (sc21733), mouse anti-human cyclin D1 monoclonal antibody (sc20044), rabbit anti- $\beta$-actin polyclonal antibody (sc130657) (dilution, 1:1000; Santa Cruz Biotechnology, Santa Cruz, CA, USA). Following incubation with the appropriate secondary antibody, the bands were visualized using enhanced chemiluminescence (Beyotime Institute of Biotechnology). The absorbance values of the target proteins and data analysis were performed using Gel-Pro Analyzer version 4.0 software (Media Cybernetics, Silver Spring, MD, USA). The protein expression levels were normalized to $\beta$-actin.

Cell proliferation analysis. The FTC133 cells were seeded at a density of $5 \times 10^{3} / 200 \mu 1$ into 96 -well microplates following pSUPER-neo-IQGAP1-siRNA transfection and G418 selection, and were then serum-starved overnight at $37^{\circ} \mathrm{C}$, and treated with $10 \mathrm{nM}$ (E2; Sigma-Aldrich, St. Louis, MO, USA) or ethanol as a vehicle (Invitrogen Life Technologies). Following culture for 24,48 and $72 \mathrm{~h}$, the target cells were added to $20 \mu \mathrm{l}$ MTT (5 mg/ml; Sigma-Aldrich) solution and incubated at $37^{\circ} \mathrm{C}$ for $4 \mathrm{~h}$. The supernatant was removed and $150 \mu \mathrm{l}$ dimethyl sulfoxide (Sigma-Aldrich) was added to each well. Following dissolving of the dark-blue MTT crystals, the absorbance was determined using a Bio-Rad microplate reader (Bio-Rad) at a wavelength of $490 \mathrm{~nm}$.

Reporter luciferase assay. Luciferase and $\beta$-galactosidase assays were performed, according to the manufacturer's instructions (Promega Corporation, Madison, WI, USA). The cultured FTC133 cells were seeded at a density of $2 \times 10^{5} /$ well into 6 -well plates and co-transfection was performed using the pSUPER-neo-IQGAP1-siRNA or pSUPER-neo-IQGAP1-siRNA-N expression vector $(1 \mu \mathrm{g})$, a reporter gene (PC3-LUC vector; $300 \mathrm{ng}$ ) and a pCMV- $\beta$ galactosidase internal control vector (150 ng; Clontech, Palo Alto, CA, USA), or as indicated in the figure legends using Lipofectamine 2000 . Following $36 \mathrm{~h}$ of transfection at $37^{\circ} \mathrm{C}$, the FTC133 cells were pretreated with E2, as described above, for $12 \mathrm{~h}$ at $37^{\circ} \mathrm{C}$ and then harvested. The reporter gene activity was obtained following normalization of the luciferase activity with that of $\beta$-galactosidase.

Cell invasion assay. The invasion assay was performed using Matrigel-coated 24-well Transwell chambers with $8.0 \mu \mathrm{m}$ polycarbonated filters (Corning Incorporated, Corning, NY, USA). Following transfection with pSUPER-neo-IQGAP1-siRNA and G418 selection, the FTC133 cells were pretreated with E2, as described above, for $12 \mathrm{~h}$, and then seeded in serum-free DMEM medium at a density of $2 \times 10^{5}$ cells $/ 300 \mu 1$ into the upper compartment, while the DMEM medium in the lower compartment was supplemented with $15 \%$ FBS. After $24 \mathrm{~h}$, the non-invasive cells on the upper surface of the membrane were removed using cotton swabs, and the invasive cells that had penetrated through the pores and migrated to the underside of the membrane were fixed using $4 \%$ paraformaldehyde and stained with $0.1 \%$ crystal violet (Sigma-Aldrich). The number of invasive cells was counted under a microscope (Olympus BX50, Tokyo, Japan).

Cell lysates and co-immunoprecipitation assay. The COS-7 and FTC133 cells were plated in 6-well plates to obtain $85 \%$ confluence at $37^{\circ} \mathrm{C}$. After $24 \mathrm{~h}$, each plate was transfected with $2 \mu \mathrm{g}$ myc-tagged pcDNA3.1-IQGAP1 plasmids and $2 \mu \mathrm{g}$ myc-tagged pcDNA3.1-ER $\alpha$ plasmids. After $48 \mathrm{~h}$, the cells were pretreated with $\mathrm{E} 2$, as described above, for $12 \mathrm{~h}$, lysed with $500 \mu$ l buffer A, which contained $50 \mathrm{mM}$ Tris $\mathrm{HCl}$ (pH 7.4), $150 \mathrm{mM} \mathrm{NaCl}, 1 \%$ Triton $\mathrm{X}-100$, protease and phosphatase inhibitors and $1 \mathrm{mM}$ PMSF. The lysates were incubated on ice for $30 \mathrm{~min}$, and insoluble material was precipitated by centrifugation at $20,000 \mathrm{x} \mathrm{g}$ for $10 \mathrm{~min}$ at $4^{\circ} \mathrm{C}$. The supernatants were pre-cleared using glutathione-sepharose beads (GE Healthcare Life Sciences, Piscataway, NJ, USA) for $1 \mathrm{~h}$ at $4^{\circ} \mathrm{C}$. Equal quantities of protein lysate were incubated with protein A-Sepharose beads (GE Healthcare Life Sciences) and either anti-rabbit IgG (\#43413; Sigma-Aldrich) or mouse anti-human IQGAP1 monoclonal antibody (BD Biosciences), or mouse anti-human ER $\alpha$ monoclonal antibody (Santa Cruz Biotechnology) overnight at $4^{\circ} \mathrm{C}$. The samples were then washed 5 times with Buffer B (Shanghai Sangon Biotechnology Co. Ltd.), containing $50 \mathrm{mM}$ Tris $\mathrm{HCl}$ (pH 7.4), $150 \mathrm{mM} \mathrm{NaCl}$ and $1 \%$ Triton $\mathrm{X}-100$, were resolved by SDS-PAGE, and were processed by SDS-PAGE and western blot analysis as described above.

Statistical analysis. The results are expressed as the mean \pm standard deviation of three individual experiments performed in triplicate. All analyses were conducted using SPSS software (SPSS Inc., Chicago, IL, USA). Statistical analysis was performed using Student's t-test and analysis of variance. ${ }^{*} \mathrm{P}<0.05$ was considered to indicate a statistically significant difference.

\section{Results}

IQGAPI is overexpressed in follicular thyroid cancer and FTC133 cells. The results of the western blotting demonstrated that the protein levels of IQGAP1 were significantly increased in the follicular thyroid cancer tissues and FTC133 cells, compared 
$\mathbf{A}$
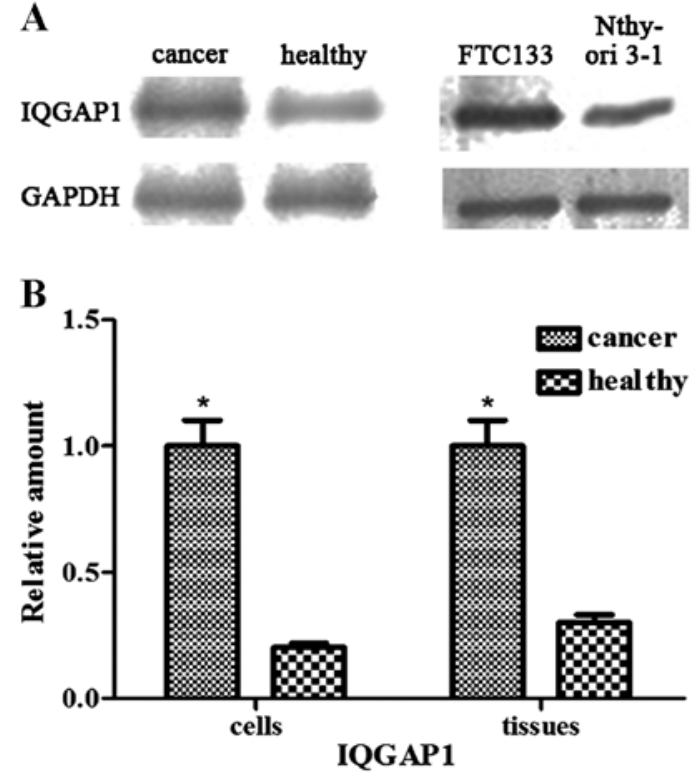

Figure 1. IQGAP1 is overexpressed in follicular thyroid cancer and FTC133 cells. (A) Protein expression of IQGAP1 in (left) follicular thyroid cancer and adjacent non-neoplastic tissue samples, and (right) FTC133 cells and Nthy-ori 3-1 cells were examined using western blotting. $\beta$-actin was used as an internal control. (B) Expression of IQGAP1 proteins were analyzed using Gel-Pro Analyzer version 4.0 software and normalized to $\beta$-actin. ${ }^{*} \mathrm{P}<0.05$, compared with adjacent tissue samples or Nthy-ori 3-1 cells. Data are expressed as the mean \pm standard deviation. IQGAP1, IQ-domain GTPase-activating protein 1

with the weak levels of expression observed in the adjacent non-neoplastic tissues and Nthy-ori 3-1 cells $(\mathrm{P}<0.05$; Fig. 1).

IQGAPI knockdown represses cell proliferation and ER $\alpha$ transcriptional activity. In the present study, an MTT assay was used to investigate whether ectopic IQGAP1 affects cell proliferation in the FTC133 thyroid cancer cell line. Compared with the FTC133 cells transfected with pSUPER-neo-IQGAP1-siRNA-N, the proliferation of the FTC133 cells transfected with pSUPER-neo-IQGAP1-siRNA was significantly reduced to $79.27,57.49$ and $55.14 \%$ at 24 , 48 and 72 h, respectively ( $<<0.05$; Fig. 2A). No significant difference was observed between the control cells and the cells transfected with pSUPER-neo-IQGAP1-siRNA-N $(\mathrm{P}>0.05)$.

In addition, the present study used the well-characterized ERE-reporter assay to examine the effect of endogenous IQGAP1 on ER $\alpha$ transcriptional activity following E2 pretreatment in FTC133 cells. The expression of IQGAP1 was effectively knocked down by pSUPER-neo-IQGAP1-siRNA plasmid transfection, which led to a significant reduction of ERE reporter activity, regardless of whether E2 treatment had been performed or not (Fig. 2B). Therefore, knockdown of the expression of IQGAP1 in FTC133 cells contributed to decreased cell proliferation and inhibited the transcriptional activity of ER $\alpha$.

IQGAPI knockdown represses the invasion of FTC133 cells. In the present study, the effect of pSUPER-neo-IQGAP1-siRNA on the protein expression levels of E-cadherin and MMP-9 were examined, which are known to be involved in cancer cell adhesion, invasion and progression. The protein levels of E-cadherin were significantly upregulated, while those of
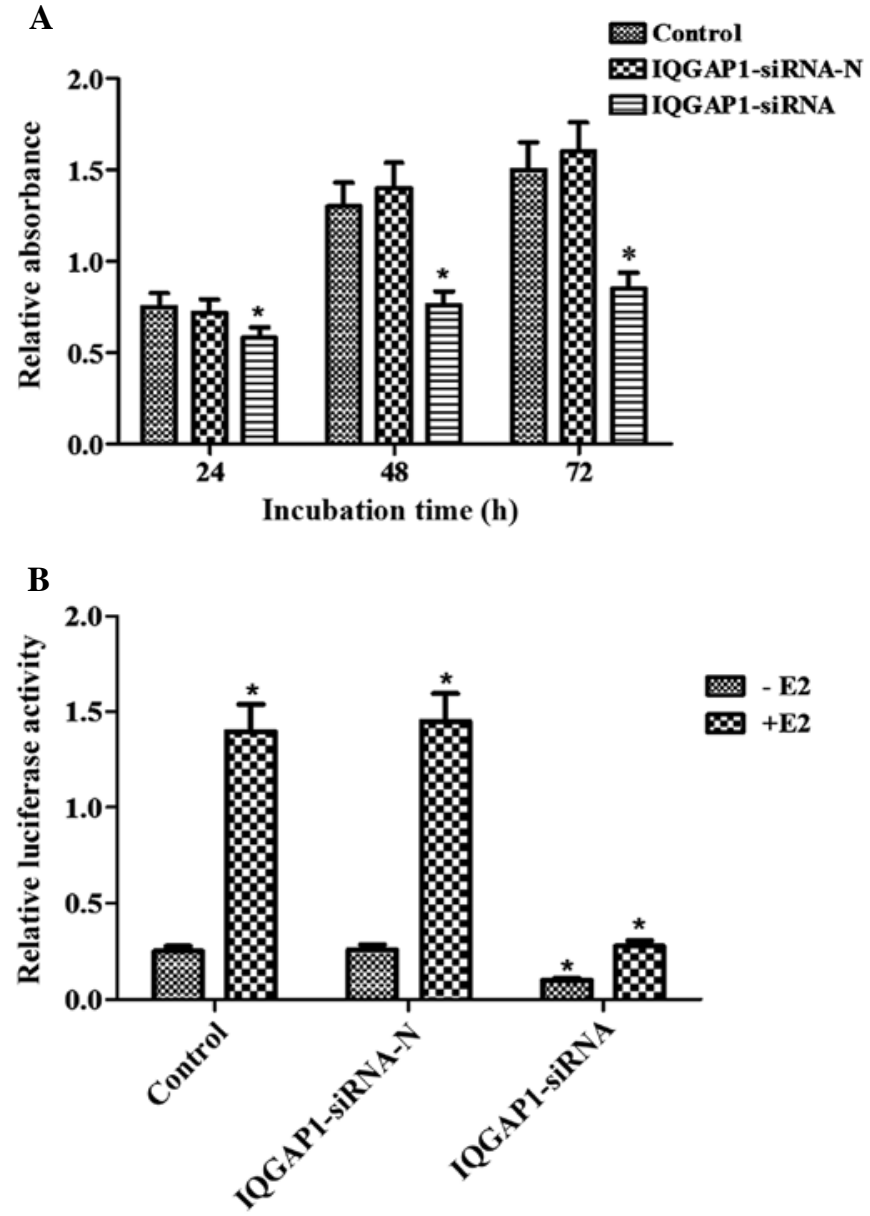

Figure 2. IQGAP1 knockdown represses cell proliferation and estrogen receptor $\alpha$ transcriptional activity. (A) Decrease in FTC133 cell proliferation following transfection with pSUPER-neo-IQGAP1-siRNA. FTC133 cells were seeded on 96-well microplates, cultured for 24,48 and $72 \mathrm{~h}$ and stained with MTT. The number of viable cells were determined via the absorbance. ${ }^{*} \mathrm{P}<0.05$, compared with control. (B) At $36 \mathrm{~h}$ post-transfection with the PC3-LUC reporter (pCMV- $\beta$ galactosidase) and indicated expression constructs, the cells were serum-starved and treated with $10 \mathrm{nM} \mathrm{E2}$ or ethanol for $12 \mathrm{~h}$. The cells were harvested and then processed for the measurement of transcriptional activity using a luciferase assay. ${ }^{*} \mathrm{P}<0.05$, compared with non-E2 stimulation or control. Data are expressed as the mean \pm standard deviation. siRNA, small interfering RNA; IQGAP1, IQ-domain GTPase-activating protein 1; E2, 17ß-estradiol..

MMP-9 were significantly downregulated following transfection with pSUPER-neo-IQGAP1-siRNA, compared with the control or the cells transfected with pSUPER-neo-IQGAP1-si RNA-N (Fig. 3A and 3B).

Matrigel invasion assays were also used for functional investigation of whether IQGAP1 is involved in thyroid cancer cell invasion. pSUPER-neo-IQGAP1-siRNA transfection markedly inhibited the invasion of FTC133 cells (Fig. 3C). Therefore, these data all confirmed the specific and important role of IQGAP1 in the invasiveness of thyroid cancer.

ERa knockdown inhibits the increases in the expression levels of $p$-ERKI/2 and cyclin DI, induced by IQGAPI overexpression. Overexpression of IQGAP1 and the ERK pathway are critical in promoting thyroid cancer cell proliferation and invasion. The present study examined whether ER $\alpha$ was involved in ERK1/2 activation and cell proliferation in the FTC133 cells. 
A

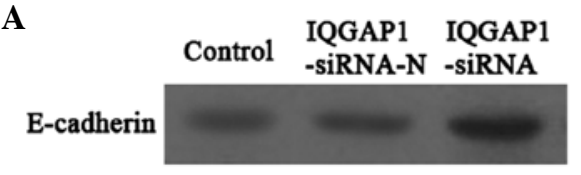

MMP-9

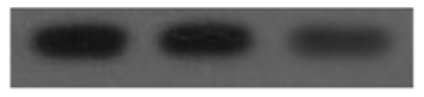

$\beta$-actin

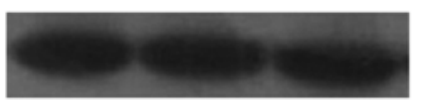

B

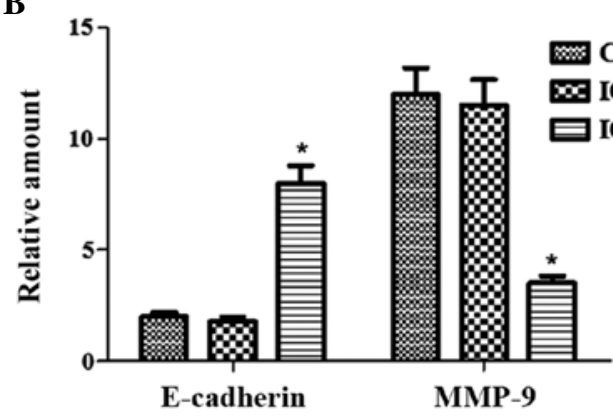

C

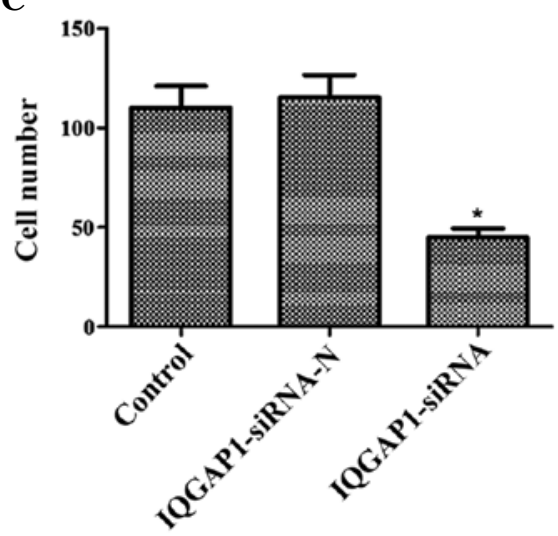

A

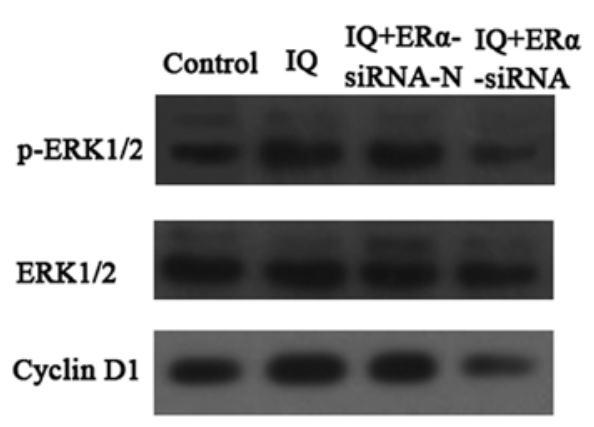

B

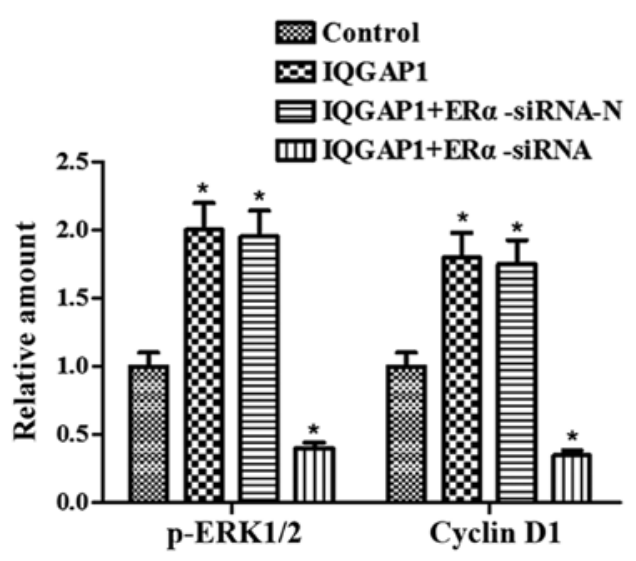

Figure 4. ER $\alpha$ knockdown downregulates the increased protein expression levels of p-ERK and cyclin D1 in FTC133 cells induced by IQGAP1 overexpression. (A) pcDNA3.1-IQGAP1, and nonspecific pSUPER-neo-ER $\alpha$-siRNA-N or specific pSUPER-neo-ER $\alpha$-siRNA were co-transfected into FTC133 cells, followed by the determination of $\mathrm{p}$-ERK1/2 and cyclin D1 proteins using western blotting. (B) Protein expression levels of p-ERK1/2, ERK1/2 and cyclin D1 were analyzed using Gel-Pro Analyzer version 4.0 software. ${ }^{*} \mathrm{P}<0.05$, compared with control. Data are expressed as the mean \pm standard deviation. siRNA, small interfering RNA. ER $\alpha$, estrogen receptor $\alpha$; IQGAP1, IQ-domain GTPase-activating protein 1; p-, phosphorylated; ERK, extracellular signal-regulated kinase.

Figure 3. IQGAP1 knockdown results in upregulation of the expression of E-cadherin, downregulation of the expression of MMP-9, and attenuates cell invasion. (A) FTC133 cells were transfected with pSUPER-neo-IQGAP1-siRNA-N or pSUPER-neo-IQGAP1-siRNA, and whole cell lysates were extracted. The E-cadherin and MMP-9 proteins were then detected using western blotting. $\beta$-actin was used as a loading control. (B) Protein expression levels of E-cadherin and MMP-9 were analyzed using Gel-Pro Analyzer version 4.0 software and normalized to $\beta$-actin (C) pSUPER-neo-IQGSP1-siRNA-transfection of FTC133 cells reduced the number of invading cells in the Transwell assay. "P<0.05, compared with control. siRNA, small interfering RNA. Data are expressed as the mean \pm standard deviation. IQGAP1, IQ-domain GTPase-activating protein 1; MMP-9, matrix metalloproteinase-9; siRNA, small interfering RNA.

The expression levels of p-ERK1/2 and cyclin D1 were upregulated following transfection with the pcDNA3.1-IQGAP1 and pSUPER-neo-ER $\alpha$-siRNA-N plasmids into the FTC133 cells (Fig. 4). By contrast, significant decreases in the protein expression of p-ERK1/2 and cyclin D1 were observed in the FTC133 cells co-transfected with pcDNA3.1-IQGAP1 and pSUPER-neo-ER $\alpha$-siRNA. These data indicated that IQGAP1 promoted activation of the ERK1/2 signaling pathway and cell proliferation, which may be inhibited by the absence of ER $\alpha$.

IQGAPI directly interacts with ER $\alpha$ in intact cells. The binding function between IQGAP1 and ER $\alpha$ has been reported in breast cancer cells (4), thus the present study used ER $\alpha$-negative COS-7

cells to detect the potential interaction between IQGAP1 and $\mathrm{ER} \alpha$ in intact cells. The co-expression of IQGAP1 and ER $\alpha$ in COS-7 cells was evaluated using an immunoprecipitation assay followed by western blot analysis. Immunoprecipitation with the anti-ER $\alpha$ or anti-IQGAP1 antibody revealed that binding of IQGAP1 to ER $\alpha$ in the COS-7 cells, with rabbit IgG considered a negative control, validating the specificity of the interaction (Fig. 5A and 5B). The interaction between IQGAP1 and endogenous ER $\alpha$ in the FTC133 cells was further examined. The cells were cross-linked using paraformaldehyde, followed by lysis. Immunoprecipitation with the anti-ER $\alpha$ antibody revealed that IQGAP1 interacted with theER $\alpha$ from the FTC133 cells, suggesting that endogenous IQGAP1 interacted directly with ER $\alpha$ in theFTC133 cells (Fig. 5C).

\section{Discussion}

The present study aimed to investigate the association between IQGAP1 and ER $\alpha$ with E2 pretreatment in the FTC133 thyroid cancer cell line. ER $\alpha$ acts as a nuclear hormone receptor, which is known to modulate the expression of genes that are implicated in different cellular processes, including tumorigenesis $(18,19)$. IQGAP1 acts as a key mediator of numerous cellular processes and signaling pathways in a broad range of interacting partners (9). 


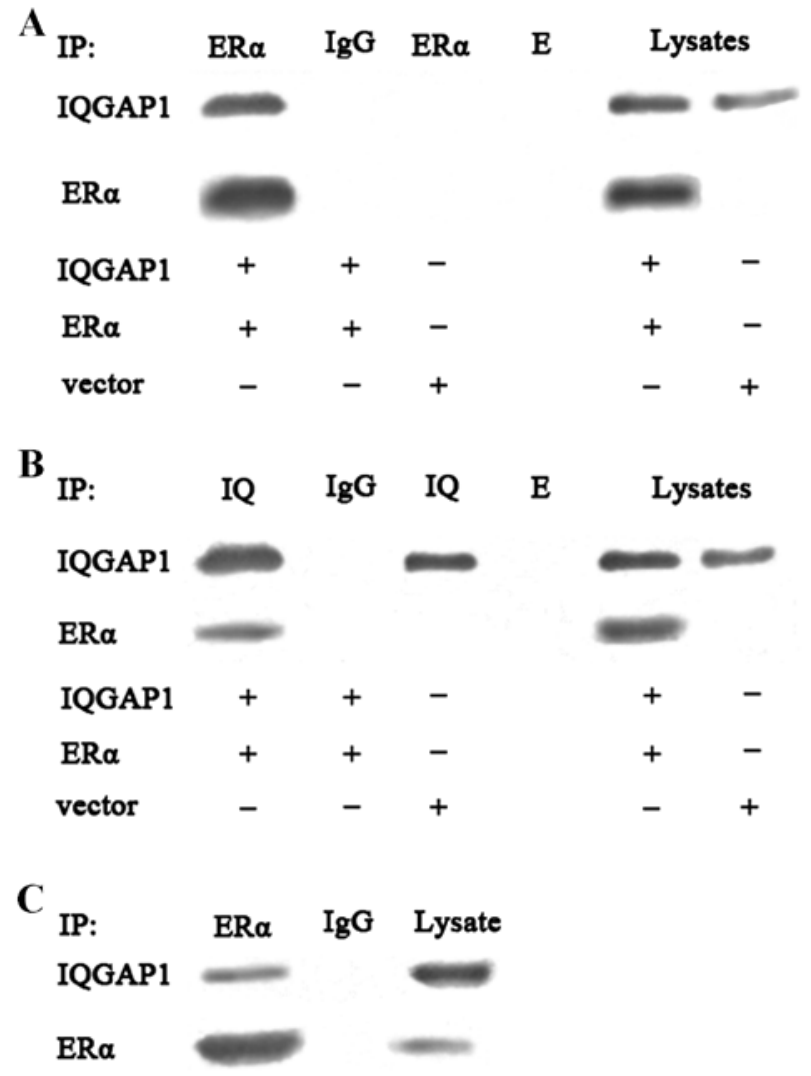

Figure 5. ER $\alpha$ and IQGAP1 co-immunoprecipitate from cell lysates. (A) COS-7 cells were transfected with IQGAP1 and ER $\alpha$ plasmids. Cell lysate proteins were detected directly (lysates) or via IP with anti-ER $\alpha$ antibodies. (B) COS-7 cells were processed, as described above, and then detected via IP with anti-IQGAP1 antibodies. (C) Non-transfected FTC133 cells were incubated with $0.2 \%$ paraformaldehyde and then processed, as described above, followed by IR with anti-ER $\alpha$ antibody. Rabbit IgG was used as a negative control. An aliquot of lysate was processed in parallel. Samples were processed using western blotting for IQGAP1 and ER $\alpha$. E, empty lane. IP, immunoprecipitation; IQGAP1/IQ, IQ-domain GTPase-activating protein 1; ER $\alpha$, Estrogen receptor $\alpha$

Western blotting revealed that IQGAP1 protein was consistently upregulated in thyroid cancer samples and FTC133 cells, compared with adjacent healthy non-neoplastic tissues and Nthy-ori 3-1 cells, as has been confirmed in other types of cancer $(9,20)$. Therefore, the selected ER $\alpha$-positive FTC133 cell line expressed high levels of IQGAP1 protein, thus enabling evaluation of the role of IQGAP1 and ER $\alpha$ in thyroid cancer cells (21). Subsequently, the effects of IQGAP1 expression and E2 stimulation on ER $\alpha$ transcriptional activity, cell proliferation, cell invasion and IQGAP1/ER $\alpha$ protein-binding were examined in the FTC133 follicular thyroid cancer cell line.

Increased evidence has indicated the connection between the expression of IQGAP1 and tumorigenesis (9), in particular the association with hormone-associated tumorigenesis, including the development of breast cancer (4). IQGAP1 can bind to ER $\alpha$ and modulate its function under the stimulation of E2 in breast cancer cells; therefore, it may be considered a therapeutic target for patients with breast carcinoma (14). However, whether the IQGAP1/ER $\alpha$ interaction is involved in thyroid cancer processes remains to be elucidated. To validate this hypothesis, a series of assays were performed in the present study by expressing knockdown, transfected or endogenous human IQGAP1 or ERa in the FTC133 cells, along with pilot investigations on tissue protein detection. This study demonstrated the IQGAP1/ER $\alpha$ interaction and its role in promoting the proliferation and invasion of human follicular thyroid cancer cells.

The present study also observedthat the knockdown of IQGAP1 by two distinct IQGAP1 siRNAs significantly inhibited proliferation of the FTC133 cells in a time-dependent manner, as is also observed in certain other types of cancer cells (22-24). Furthermore, the present study examined the possible role of IQGAP1 in E2-induced or non-E2-induced $\mathrm{ER} \alpha$ transcriptional activation using a reporter luciferase assay $(25,26)$. The results suggested that ER $\alpha$ transcriptional activation was significantly increased by E2 stimulation in the FTC133 cells and was significantly decreased in the cells, in which IQGAP1 was specifically knocked down by siRNA, in the E2 group and non-E2 group. Therefore, the possible mechanism may be that the interaction between IQGAP1 and $\mathrm{ER} \alpha$ in FTC133 cells is regulated, to a certain extent, by E2 stimulation, and that the ability of E2 to induce transcriptional activity is impaired in cells following IQGAP1 knockdown. It was hypothesized that knockdown of IQGAP1 results in a loss of cell proliferation and the E2-induced activation of ER $\alpha$ transcriptional activity in FTC133 cells.

The expression of MMP-9, which is known to promote and enhance metastasis, has been reported to correlate with E2-ER signaling $(27,28)$. Furthermore, MMP-9 secretion is increased in ER-positive thyroid cancer cells following E2 treatment (29). The abnormal expression of E-cadherin has been reported in the majority of types of human cancer, including thyroid cancer (30), and is found to correlate with the invasion and metastasis of epithelial tumor cells (31). IQGAP1 is a multifunctional protein involved in actin cytoskeleton assembly, which may be involved in cancer invasion (32-34) and in regulating E-cadherin-mediated cell-cell adhesion (35), particualrly in thyroid cancer cells (14). In the present study, IQGAP1 knockdown significantly affected the expression levels of these two adhesion-associated proteins, decreasing MMP-9 and increasing E-cadherin, and inhibited the invasion of the E2 pretreated FTC133 cells.

Previous studies have demonstrated that activation of ERK1/2 signaling is coupled with the upregulation of specific target transcription factors under E2 stimulation, for example, cyclic AMP response element-binding protein (36), a nuclear hormone receptor/estrogen receptor (37) that modulates the expression of B-cell lymphoma-2 or cyclin D1 and may be involved in cell cycle progression and cell proliferation $(36,37)$. Notably, the present study found that ER $\alpha$ knockdown negatively acted on the connection between the ERK signaling pathway and expression of cyclin D1 in the FTC133 cells though the overexpression of IQGAP1. These results indicated that IQGAP1 and ER $\alpha$ were involved in the ERK pathway and regulated the expression of cyclin D1. In addition, ER-dependent cyclin D1 transcription and DNA synthesis are considered to be downstream targets of E2-induced ERK activation $(38,39)$. These data suggested that the interaction between the IQGAP1/ $\mathrm{ER} \alpha$ proteins enhanced the ER $\alpha$-mediated transactivation activity of cyclin D1 and p-ERK in the E2-mediating signaling pathway, and that IQGAP1/ER $\alpha$ proteins may be essential in the ERK pathway and in cell proliferation in thyroid cancer.

Further in vitro analysis is required to confirm the protein interactions between IQGAP1 and nuclear receptors. In the 
present study this protein interaction was identified in whole structural domains in a complex from cell lysates using co-immunoprecipitation to validate a direct association in COS-7 cells. The results demonstrated that IQGAP1 was able to bind to ER $\alpha$ distinctly, whether endogenous or expressed, with E2 pretreatment from FTC133 cells and co-transfected COS-7 cells, respectively. In addition, the binding between intracellular IQGAP1 and ER $\alpha$ was enhanced by co-immunoprecipitation.

Using the above approaches, the present study successfully demonstrated that IQGAP1 was able to bind to endogenous or expressed ER $\alpha$ and that IQGAP1 knockdown affected ER $\alpha$ transcriptional activity, cell proliferation and invasion in the FTC133 cells. In addition, the interaction of IQGAP1 and $\mathrm{ER} \alpha$ promoted the association of ERK1/2 with the IQGAP1 molecule, which stimulated ERK1/2 phosphorylation and cyclin D1 activation. These data offer novel insight into the involvement of IQGAP1 and ER $\alpha$ in the progression of thyroid cancer. Further analyses are required to determine whether the IQGAP1/ER $\alpha$ interaction identified in the present study is involved in other cancer processes, which may provide an effective target for cancer prevention and therapy.

\section{References}

1. Kumar A, Klinge CM and Goldstein RE: Estradiol-induced proliferation of papillary and follicular thyroid cancer cells is mediated by estrogen receptors $\alpha$ and $\beta$. Int $\mathrm{J}$ Oncol 36: 1067-1080, 2010.

2. Thomas C and Gustafsson JA: The different roles of ER subtypes in cancer biology and therapy. Nat Rev Cancer 11: 597-608, 2011

3. Heldring N, Pike A, Andersson S, et al: Estrogen receptors: how do they signal and what are their targets. Physiol Rev 87: 905-931, 2007.

4. Erdemir HH, Li Z and Sacks DB: IQGAP1 binds to estrogen receptor- $\alpha$ and modulates its function. J Biol Chem 289: 9100-9112, 2014.

5. Brown MD and Sacks DB: IQGAP1 in cellular signaling: bridging the GAP. Trends Cell Biol 16: 242-249, 2006.

6. Machesky LM: Cytokinesis: IQGAPs find a function. Curr Biol 8: R202-R205, 1998.

7. Noritake J, Watanabe T, Sato K, Wang S and Kaibuchi K: IQGAP1: a key regulator of adhesion and migration. J Cell Sci 118: 2085-2092, 2005.

8. White CD, Erdemir HH and Sacks DB: IQGAP1 and its binding proteins control diverse biological functions. Cell Signal 24: 826-834, 2012.

9. Johnson M, Sharma M and Henderson BR: IQGAP1 regulation and roles in cancer. Cell Signal 21: 1471-1478, 2009.

10. White CD, Brown MD and Sacks DB: IQGAPs in cancer: a family of scaffold proteins underlying tumorigenesis. FEBS Lett 583: 1817-1824, 2009.

11. Sugimoto N, Imoto I, Fukuda Y, et al: IQGAP1, a negative regulator of cell-cell adhesion, is upregulated by gene amplification at 15q26 in gastric cancer cell lines HSC39 and 40A J Hum Genet 46: 21-25, 2001.

12. Nabeshima $K$, Shimao $Y$, Inoue $T$ and Koono $M$ : Immunohistochemical analysis of IQGAP1 expression in human colorectal carcinomas: its overexpression in carcinomas and association with invasion fronts. Cancer Lett 176: 101-109, 2002.

13. Dong P, Nabeshima K, Nishimura N, et al: Overexpression and diffuse expression pattern of IQGAP1 at invasion fronts are independent prognostic parameters in ovarian carcinomas. Cancer Lett 243: 120-127, 2006.

14. Liu Z, Liu D, Bojdani E, El-Naggar AK, Vasko V and Xing M IQGAP1 plays an important role in the invasiveness of thyroid cancer. Clin Cancer Res 16: 6009-6018, 2010.

15. Chen Z, Gibson TB, Robinson F, et al: MAP kinases. Chem Rev 101: 2449-2476, 2001

16. Schaeffer HJ and Weber MJ: Mitogen-activated protein kinases: specific messages from ubiquitous messengers. Mol Cell Biol 19: 2435-2444, 1999.
17. Thrane S, Lykkesfeldt AE, Larsen MS, Sorensen BS and Yde CW: Estrogen receptor $\alpha$ is the major driving factor for growth in tamoxifen-resistant breast cancer and supported by HER/ERK signaling. Breast Cancer Res Treat 139: 71-80, 2013.

18. Walter P, Green S, Greene G, et al: Cloning of the human estrogen receptor cDNA. Proc Natl Acad Sci USA 82: 7889-7893, 1985.

19. Kuiper GG, Enmark E, Pelto-Huikko M, Nilsson S and Gustafsson JA: Cloning of a novel receptor expressed in rat prostate and ovary. Proc Natl Acad Sci USA 93: 5925-5930, 1996.

20. Wang XX, Li XZ, Zhai LQ, Liu ZR, Chen XJ and Pei Y: Overexpression of IQGAP1 in human pancreatic cancer. Hepatobiliary Pancreat Dis Int 12: 540-545, 2013.

21. Mirebeau-Prunier D, Le Pennec S, Jacques C, et al: Estrogen-related receptor alpha and PGC-1-related coactivator constitute a novel complex mediating the biogenesis of functional mitochondria. FEBS J 277: 713-725, 2010.

22. Jadeski L, Mataraza JM, Jeong HW, Li Z and Sacks DB: IQGAP1 stimulates proliferation and enhances tumorigenesis of human breast epithelial cells. J Biol Chem 283: 1008-1017, 2008.

23. Ma Y, Jin Z, Huang J, et al: IQGAP1 plays an important role in the cell proliferation of multiple myeloma via the MAP kinase (ERK) pathway. Oncol Rep 30: 3032-3038, 2013.

24. Wang XX, Wang K, Li XZ, et al: Targeted knockdown of IQGAP1 inhibits the progression of esophageal squamous cell carcinoma in vitro and in vivo. PLoS One 9: e96501, 2014.

25. Safe S: Transcriptional activation of genes by 17 beta-estradiol through estrogen receptor-Sp1 interactions. Vitam Horm 62: 231-252, 2001.

26. Cavarretta IT, Mukopadhyay R, Lonard DM, et al: Reduction of coactivator expression by antisense oligodeoxynucleotides inhibits ERalpha transcriptional activity and MCF-7 proliferation. Mol Endocrinol 16: 253-270, 2002.

27. Nilsson UW, Garvin S and Dabrosin C: MMP-2 and MMP-9 activity is regulated by estradiol and tamoxifen in cultured human breast cancer cells. Breast Cancer Res Treat 102: 253-261, 2007.

28. Kousidou OC, Berdiaki A, Kletsas D, et al: Estradiol-estrogen receptor: a key interplay of the expression of syndecan-2 and metalloproteinase-9 in breast cancer cells. Mol Oncol 2: 223-232, 2008.

29. Rajoria S, Suriano R, George A, et al: Estrogen induced metastatic modulators MMP-2 and MMP-9 are targets of 3,3'-diindolylmethane in thyroid cancer. PLoS One 6: e15879, 2011.

30. Graff JR, Greenberg VE, Herman JG, et al: Distinct patterns of E-cadherin $\mathrm{CpG}$ island methylation in papillary, follicular, Hurthle's cell, and poorly differentiated human thyroid carcinoma. Cancer Res 58: 2063-2066, 1998.

31. Shiozaki H, Oka H, Inoue M, Tamura S and Monden M: E-cadherin mediated adhesion system in cancer cells. Cancer 77: 1605-1613, 1996.

32. Mataraza JM, Briggs MW, Li Z, Entwistle A, Ridley AJ and Sacks DB: IQGAP1 promotes cell motility and invasion. J Biol Chem 278: 41237-41245, 2003.

33. Watanabe T, Wang S, Noritake J, et al: Interaction with IQGAP1 links APC to Rac1, Cdc42, and actin filaments during cell polarization and migration. Dev Cell 7: 871-883, 2004.

34. Yamaoka-Tojo M, Ushio-Fukai M, Hilenski L, et al: IQGAP1, a novel vascular endothelial growth factor receptor binding protein, is involved in reactive oxygen species - dependent endothelial migration and proliferation. Circ Res 95: 276-283, 2004.

35. Fukata M, Kuroda S, Nakagawa M, et al: Cdc42 and Rac1 regulate the interaction of IQGAP1 with beta-catenin. J Biol Chem 274: 26044-26050, 1999.

36. Wu TW, Wang JM, Chen S and Brinton RD: 17Beta-estradiol induced $\mathrm{Ca}^{2+}$ influx via L-type calcium channels activates the Src/ERK/cyclic-AMP response element binding protein signal pathway and BCL-2 expression in rat hippocampal neurons: a potential initiation mechanism for estrogen-induced neuroprotection. Neuroscience 135: 59-72, 2005.

37. Acconcia F, Ascenzi P, Bocedi A, et al: Palmitoylation-dependent estrogen receptor alpha membrane localization: regulation by 17beta-estradiol. Mol Biol Cell 16: 231-237, 2005.

38. Marino M, Acconcia F, Bresciani F, Weisz A and Trentalance A: Distinct nongenomic signal transduction pathways controlled by 17beta-estradiol regulate DNA synthesis and cyclin $\mathrm{D}(1)$ gene transcription in HepG2 cells. Mol Biol Cell 13: 3720-3729, 2002.

39. Marino M, Acconcia F and Trentalance A: Biphasic estradiol-induced AKT phosphorylation is modulated by PTEN via MAP kinase in HepG2 cells. Mol Biol Cell 14: 2583-2591, 2003. 